\title{
Circuit-Based Model Order Reduction for EM-CAD
}

\author{
Valentín de la Rubia and Sofía Tinoco-Galafate
}

\begin{abstract}
In this work, we focus on obtaining an accurate and reliable physical-based description of the Electromagnetics we are dealing with in order to carry out a fast and accurate computer-aided design. Appropriate Finite Element solution to time-harmonic Maxwell's equations in the frequency band of interest is involved and a reliable reduced-order model is obtained. Then, the electromagnetic behavior in the microwave device is rearranged and described in terms of circuit theory, giving rise to the coupling matrix representation of the original Maxwell problem.
\end{abstract}

Index Terms-Circuit theory, computer aided engineering, error analysis, finite element methods, Galerkin method, reduced basis methods, reduced order systems.

\section{INTRODUCTION}

In this work, we focus on a rapid and reliable frequencyparameter sweep in microwave circuits based on the reducedbasis method [1], [2] and we place especial attention in getting valuable circuit theory information out of the reliable reduced-order model. The proposed model order reduction is based upon the following observation: should the field solution of the original system be considered as a function of frequency, the field solution itself does not arbitrarily vary as frequency changes, but it varies smoothly with frequency, i.e., the field solution resides on a very low dimensional subspace induced by the frequency variation. The reducedbasis method focuses on approximating the evolution of the electromagnetic field as a function of frequency, rather than approximating the electromagnetic field itself as the FEM does. We propose a model order reduction approach where field solutions at different frequencies are used as new basis functions to capture the dynamics of the electromagnetic field as frequency changes. Galerkin projection is applied to reduce the original FEM system into a small system detailing the frequency behavior of the original Maxwell problem.

\section{PRoblem STATEMENT}

Throughout this work, we deliberately refer to the wavenumber $k$ as frequency for convenience. Thus, given a frequency band of interest, namely, $\left[k_{\min }, k_{\max }\right]$, the FEM problem we aim at solving results in the following linear system of equations after discretization:

$$
\begin{aligned}
& \text { Find } \mathbf{x}(k) \text { such that: } \\
& \mathbf{A}(k) \mathbf{x}(k)=\mathbf{b}(k)
\end{aligned}
$$

where $\mathbf{A}(k)$ is a $\mathcal{N} \times \mathcal{N}$ matrix operator, $\mathbf{x}(k)$ and $\mathbf{b}(k)$ are the field solution and forcing term vectors, respectively. Making explicit the frequency dependence in the matrix operator and the right hand side, the following structure arises whenever homogeneous waveguide ports are taken into account:

$$
\begin{gathered}
\mathbf{A}(k)=\mathbf{S}-k^{2} \mathbf{T} \\
\mathbf{b}(k)=f(k) \mathbf{b},
\end{gathered}
$$

where $\mathbf{S}$ and $\mathbf{T}$ are the stiffness and mass matrices and $f(k)$ is an analytic function of frequency [1].

\section{A. Model Order Reduction}

The reduced-order model that we propose draws upon using field solutions at specific frequencies as basis functions to represent the evolution of the electromagnetic field as a function of frequency. Thus, we carry out Galerkin projection onto the space $\mathcal{W}^{N}=\operatorname{span}\left\{\zeta_{\mathbf{1}} \equiv \mathbf{x}\left(k_{1}\right), \ldots, \zeta_{\mathbf{N}} \equiv \mathbf{x}\left(k_{N}\right)\right\}$, where $k_{1}, \ldots, k_{N} \in\left[k_{\min }, k_{\max }\right]$, to obtain the following reduced system:

$$
\begin{aligned}
& \text { Find } \widetilde{\mathbf{x}}(k) \text { such that: } \\
& \widetilde{\mathbf{A}}(k) \widetilde{\mathbf{x}}(k)=\widetilde{\mathbf{b}}(k)
\end{aligned}
$$

where $\widetilde{\mathbf{A}}(k)$ is the reduced $N \times N$ matrix operator $(N \ll \mathcal{N})$, $\widetilde{\mathbf{x}}(k)$ is the reduced solution coefficient vector and $\widetilde{\mathbf{b}}(k)$ is the reduced forcing term. So far,

$$
\begin{gathered}
\widetilde{\mathbf{A}}(k)=\widetilde{\mathbf{S}}-k^{2} \widetilde{\mathbf{T}} \\
\widetilde{\mathbf{b}}(k)=f(k) \widetilde{\mathbf{b}},
\end{gathered}
$$

where $\widetilde{\mathbf{S}}=\mathbf{P}^{T} \mathbf{S P}, \widetilde{\mathbf{T}}=\mathbf{P}^{T} \mathbf{T} \mathbf{P}$ and $\widetilde{\mathbf{b}}=\mathbf{P}^{T} \mathbf{b}, \mathbf{P}=$ $\operatorname{cols}\left\{\zeta_{\mathbf{1}}, \ldots, \zeta_{\mathbf{N}}\right\}$. Finally, the field solution is obtained with ease as $\mathbf{x}(k) \simeq \mathbf{P} \widetilde{\mathbf{x}}(k)=\sum_{n=1}^{N} \widetilde{x}_{n}(k) \zeta_{\mathbf{n}}$.

We refer to [1] for a detailed explanation on the actual automatic adaptive selection of the frequency samples $k_{1}, \ldots, k_{N}$ $\in\left[k_{\min }, k_{\max }\right]$ while certifying the accuracy of the reduced order model. As a result, a fully automatic and reliable model order reduction is carried out.

\section{B. Circuit Theory Representation in Electromagnetics}

In the reduced-order model (3), an eigendecomposition is carried out to write down the reduced-order electromagnetic problem in terms of global eigenresonances or eigenmodes. Thus, (3) reads

$$
\begin{gathered}
\text { Find } \widetilde{\mathbf{x}}(k) \text { such that: } \\
\left(\boldsymbol{\Lambda}-k^{2} \mathbf{I}\right) \mathbf{V} \widetilde{\mathbf{x}}(k)=f(k) \mathbf{V} \widetilde{\mathbf{T}}^{-1} \widetilde{\mathbf{b}} \text { for } k \in\left[k_{\min }, k_{\max }\right],
\end{gathered}
$$

where $\widetilde{\mathbf{T}}^{-1} \widetilde{\mathbf{S}}=\mathbf{V}^{-\mathbf{1}} \mathbf{\Lambda} \mathbf{V}, \boldsymbol{\Lambda}$ is a diagonal matrix and $\mathbf{I}$ is the identity matrix. It should be noted that a change of basis has been applied. This matrix representation gives rise to a coupling matrix in transversal topology introduced 


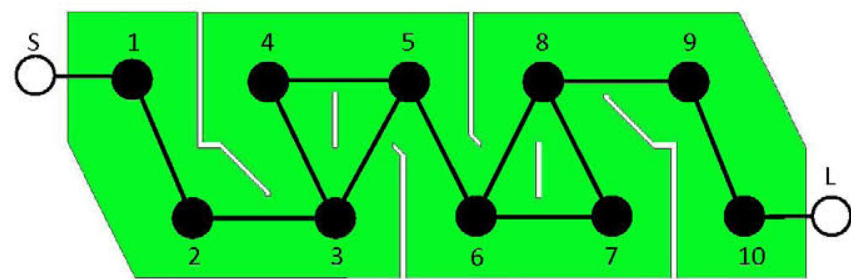

Fig. 1. $10^{\text {th }}$ order $1800 \mathrm{MHz}$ ceramic TM mode filter. Intended coupling topology.

by Cameron in microwave filters [3] once the Generalized Admittance Matrix is computed in this problem, cf. [1]. As a result, we have a circuit representation of the electromagnetic behavior by means of a rigorous solution of the Maxwell problem (1). In order to obtain further physical insight of the microwave device, a coupling matrix representation of the Electromagnetics for the actual coupling topology in the microwave device layout is obtained by applying the technique proposed in [4]. This serves for electromagnetic design and diagnosis purposes since all electromagnetic phenomena are taking into account.

\section{NUMERICAL RESULTS}

In this section, we apply the proposed reliable circuit model for electromagnetic diagnosis in a $10^{\text {th }}$ order $1800 \mathrm{MHz}$ ceramic TM mode filter with two transmission zeros. See Fig. 1, where the intended coupling topology is highlighted. The purpose in here is to understand the actual electromagnetic coupling behavior among the different resonators in the structure. The 1.6-1.9 GHz band is taken into account, which give rise to a reduced-order model of dimension 14, namely 14 eigenmodes are present. Among these 14 eigenmodes described in the reduced-order model, 10 correspond to dominant global resonances (the order of the filter), whereas the remaining 4 stand for parasitics behavior in electromagnetics. The latter will be taken into account as a direct source to load coupling in the coupling matrix representation. Once this technique is applied to this filter, the coupling matrix described in equation (6) is obtained. This coupling matrix reveals that leakage couplings are present between resonators 1 and 3 , and between resonators 8 and 10. See Fig. 2. These additional couplings are the result of Electromagnetics. However, not only this technique is able to predict the existence of additional unwanted couplings, but also their actual location within the circuit layout and their corresponding coupling values. This information is extremely helpful not only for diagnosis but also in the design process. Having a reliable circuit representation of electromagnetic behavior allows a better understanding of the physics in microwave engineering.

\section{CONCLUSION}

A reduced-order model in FEM analysis has been detailed. Special emphasis has been placed in determining a circuit theory representation within the reduced-order model in FEM analysis. The proposed technique has been applied for electromagnetic diagnosis in microwave devices. A coupling matrix representation, namely, a circuit theory representation, of electromagnetic phenomena has given rise to a better understanding for CAD in Maxwell problems.

$$
\left(\begin{array}{cccccccccccc}
0 & 1.03 & 0 & 0 & 0 & 0 & 0 & 0 & 0 & 0 & 0 & 0 \\
1.03 & -0.53 & 0.82 & 0.01 & 0 & 0 & 0 & 0 & 0 & 0 & 0 & 0 \\
0 & 0.82 & 0.01 & 0.63 & 0 & 0 & 0 & 0 & 0 & 0 & 0 & 0 \\
0 & 0.01 & 0.63 & 0.02 & 0.56 & 0.24 & 0 & 0 & 0 & 0 & 0 & 0 \\
0 & 0 & 0 & 0.56 & -0.41 & 0.53 & 0 & 0 & 0 & 0 & 0 & 0 \\
0 & 0 & 0 & 0.24 & 0.53 & 0.02 & 0.52 & 0 & 0 & 0 & 0 & 0 \\
0 & 0 & 0 & 0 & 0 & 0.52 & 0.03 & 0.55 & 0.20 & 0 & 0 & 0 \\
0 & 0 & 0 & 0 & 0 & 0 & 0.55 & -0.51 & 0.54 & 0 & 0 & 0 \\
0 & 0 & 0 & 0 & 0 & 0 & 0.20 & 0.54 & 0.02 & 0.65 & 0.01 & 0 \\
0 & 0 & 0 & 0 & 0 & 0 & 0 & 0 & 0.65 & 0.21 & 0.83 & 0 \\
0 & 0 & 0 & 0 & 0 & 0 & 0 & 0 & 0.01 & 0.83 & -0.39 & 1.03 \\
0 & 0 & 0 & 0 & 0 & 0 & 0 & 0 & 0 & 0 & 1.03 & 0
\end{array}\right) .
$$

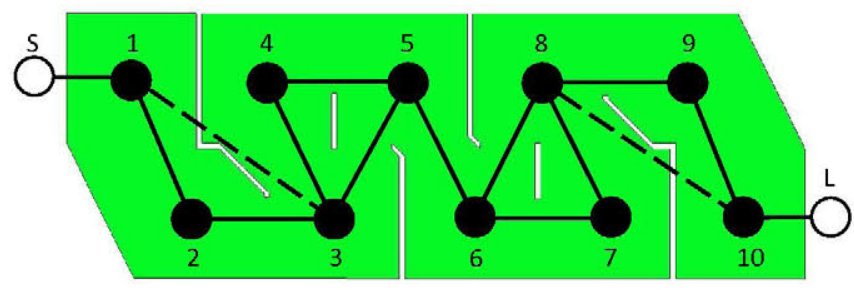

\section{REFERENCES}

[1] V. de la Rubia, U. Razafison, and Y. Maday, "Reliable fast frequency sweep for microwave devices via the reduced-basis method," IEEE Trans. Microwave Theory Tech., vol. 57, no. 12, pp. 2923-2937, Dec. 2009.

[2] A. Quarteroni, A. Manzoni, and F. Negri, Reduced Basis Methods for Partial Differential Equations: An Introduction. Springer, 2016, vol. 92.

[3] R. J. Cameron, "Advanced coupling matrix synthesis techniques for microwave filters," IEEE Trans. Microwave Theory Tech., vol. 51, no. 1, pp. 1-10, Jan. 2003.

[4] P. Kozakowski and M. Mrozowski, "Quadratic programming approach to coupled resonator filter cad," IEEE Trans. Microwave Theory Tech., vol. 54, no. 11, pp. 3906-3913, Nov. 2006.

Fig. 2. $10^{\text {th }}$ order $1800 \mathrm{MHz}$ ceramic TM mode filter. Actual coupling topology. 